\title{
Development of Cymbidium ensifolium genic-SSR markers and their utility in genetic diversity and population structure analysis in cymbidiums
}

\author{
Xiaobai Li $i^{*}$, Feng Jin², Liang Jin ${ }^{1}$, Aaron Jackson ${ }^{3}$, Cheng Huang ${ }^{4}$, Kehu Li ${ }^{5}$ and Xiaoli Shu ${ }^{6 *}$
}

\begin{abstract}
Background: Cymbidium is a genus of 68 species in the orchid family, with extremely high ornamental value. Marker-assisted selection has proven to be an effective strategy in accelerating plant breeding for many plant species. Analysis of cymbidiums genetic background by molecular markers can be of great value in assisting parental selection and breeding strategy design, however, in plants such as cymbidiums limited genomic resources exist. In order to obtain efficient markers, we deep sequenced the $C$. ensifolium transcriptome to identify simple sequence repeats derived from gene regions (genic-SSR).
\end{abstract}

Result: The 7,936 genic-SSR markers were identified. A total of 80 genic-SSRs were selected, and primers were designed according to their flanking sequences. Of the 80 genic-SSR primer sets, 62 were amplified in C. ensifolium successfully, and 55 showed polymorphism when cross-tested among 9 Cymbidium species comprising 59 accessions. Unigenes containing the 62 genic-SSRs were searched against Non-redundant (Nr), Gene Ontology database (GO), eukaryotic orthologous groups (KOGs) and Kyoto Encyclopedia of Genes and Genomes (KEGG) database. The search resulted in 53 matching $\mathrm{Nr}$ sequences, of which 39 had GO terms, 18 were assigned to KOGs, and 15 were annotated with KEGG. Genetic diversity and population structure were analyzed based on 55 polymorphic genic-SSR data among 59 accessions. The genetic distance averaged 0.3911 , ranging from 0.016 to 0.618 . The polymorphic index content (PIC) of 55 polymorphic markers averaged 0.407 , ranging from 0.033 to 0.863 . A model-based clustering analysis revealed that five genetic groups existed in the collection. Accessions from the same species were typically grouped together; however, C. goeringii accessions did not always form a separate cluster, suggesting that C. goeringii accessions were polyphyletic.

Conclusion: The genic-SSR identified in this study constitute a set of markers that can be applied across multiple Cymbidium species and used for the evaluation of genetic relationships as well as qualitative and quantitative trait mapping studies. Genic-SSRs coupled with the functional annotations provided by the unigenes will aid in mapping candidate genes of specific function.

Keywords: Cymbidium ensifolium, Genic-SSR, Genetic diversity, Population structure

\section{Background}

Cymbidium is a genus of 68 species in the orchid family [1]. Cymbidium species are mainly distributed in the tropical and subtropical regions of Asia, including northwest India, China, Japan, Korea, the Malay Archipelago, and north and east Australia [2,3]. A total of 49 species

\footnotetext{
*Correspondence: hufanfan1982815@gmail.com; shuxl@zju.edu.cn 'Zhejiang Academy of Agricultural Sciences, Shiqiao Road 139, Hangzhou 310021, People s Republic of China

${ }^{6}$ State Key Lab of Rice Biology, International Atomic Energy Agency Collaborating Center, Zhejiang University, Hangzhou 310029, Peoples Republic of China Full list of author information is available at the end of the article
}

can be found in China, including five famous species, i.e., C. goeringii, C. faberi, C. ensifolium, C. kanran, and $C$. sinense. These cymbidiums comprise some of the rarest plant species, with only a few surviving original populations and some reintroduced plants in the south of China, including Yunnan and Taiwan [4]. The fascinating varieties and shapes of their flowers endow these species with extremely high ornamental value that has attracted the worlds attention. 
Knowledge of the genetic diversity and population structure of germplasm collections is an important foundation for plant improvement [5]. Estimation of genetic distance among germplasm is helpful in selecting parental combinations for creating segregating populations so as to maintain genetic diversity in a breeding program. However, genetic diversity may appear spatially structured at different scales, such as population, subpopulation or among neighboring individuals [6]. Population genetic analyses can provide important parameters including standing levels of genetic variation and the partitioning of this variability within/ between populations [7]. The genetic diversity or population structure of C. ensifolium and other cymbidiums have been measured by using different molecular tools, including restriction enzyme polymorphism (RFLP) markers [3], random amplified polymorphic DNA (RAPD) markers $[3,4,8]$, amplified fragment length polymorphism (AFLP) markers [4], polymorphisms of internal transcribed spacers (ITS) of nuclear ribosomal DNA and plastid, inter-simple sequence repeats (ISSR) markers [4,9], and SSRs [10,11]. Compared with RAPD, ISSR and ITS, SSR markers are more reliable, locus-specific, codominant, highly polymorphic, and well distributed throughout the genome [12]. Moreover, SSR s only require polymerase chain reaction (PCR), which is a big advantage over RFLP and AFLP. These features make SSR s well suited for marker-assisted selection, genetic diversity analysis, population genetic analysis, genetic mapping, and genetic map comparison in various species $[13,14]$.

The number of SSR is very limited for $C$. ensifolium, due to limited sequence resources. Until now, the National Center for Biotechnology Information (NCBI) contained very limited Cymbidium sequence information, i.e., 692 nucleotide sequences and 78 expressed sequence tags (ESTs) (http://www.ncbi.nlm.nih.gov/nucest?term=cymbidium\%5BOrganism\%5D, verified 2014). RNA-seq provides a fast, cost-effective, and reliable approach for generating large-scale transcriptome data in non-model species, and also offers an opportunity to identify and develop genicSSRs by transcriptome data mining [15]. Compared with traditional anonymous SSRs from genomic DNA, these new genic-SSR markers have two advantages, i.e. a wealth of functional annotations and high transferability across taxa $[15,16]$. Herein, we extracted the total mRNA from C. ensifolium flower buds for RNA-seq, which resulted in $9.52 \mathrm{~Gb}$ of transcriptome data. From the C.ensifloium transcriptome, we obtained 55 new polymorphic microsatellite loci after testing their transferability across 59 Cymbidium accessions.

\section{Methods}

\section{Plant materials}

A total of $11 \mathrm{C}$. ensifolium accessions were employed to test genic-SSRs and additional 47 accessions from
C. lancifolium, C. floribundum, C. suavissimum, C. cyperifolium, C. qiubeiense, C. faberi, C. goeringii and C. sinense were used to cross-test these markers among multiple species. The plants were grown and maintained in a greenhouse at the Zhejiang University under natural light (Table 1). Fresh leaf samples were collected from two or three seedling of each accession for genomic DNA extraction.

\section{Genic-SSR search and primer design}

Total RNA was isolated from native cultivar of C. ensifolium Tiegusu using TRIzol? reagent (Invitrogen, CA, USA) and treated with RNase-free DNase I (TaKaRa Bio, Dalian, China) for 45 min according to the manufacturer $\mathrm{s}$ protocol. The RNA was used in cDNA library construction and Illumina deep sequencing [17]. The raw sequencing reads were stringently filtered, and high-quality reads were assembled de novo using Trinity with an optimized k-mer length of 25 [18]. MSATCOMMANDER V. 0.8.2 [19] was used to analyze SSR distribution. The minimum number of repeats for SSR detection was as follows: six for di-SSRs, and four for tri-, tetra-, penta-, and hexa-SSRs. The open reading frame (ORF) and untranslated region (UTR) within unigenes were identified using Trinity [18]. Software Primer3.0 [20] was used to design primers for genic-SSR loci with sufficient flanking sequences.

Unigenes containing genic-SSRs were compared with protein databases, including the non-redundant $(\mathrm{Nr})$ database (http://www.ncbi.nlm.nih.gov/), using BLASTX with a significance cut-off $E$-value of $1 \mathrm{e}^{-5}$ [17]. For the non-redundant annotations, BLAST2GO V. 2.4.4 was used to obtain Gene Ontology (GO) annotations of unique transcripts [21]. Metabolic pathway analysis were performed based on the pathways of Oryza sativa in the Kyoto Encyclopedia of Genes and Genomes (KEGG) $[22,23]$. The unigene sequences were also aligned to the KOG (Eukaryotic Orthologous Groups) database to predict and classify possible functions [24].

\section{Genotyping}

Genomic DNA was extracted from leaf samples as previously described [25]. PCR primers were synthesized by Life Technologies (AB \& Invitrogen, Shanghai, China). PCR reactions were conducted based on a previously published protocol [26]. The PCR products were separated through polyacrylamide gel electrophoresis using $8 \%$ bis-acrylamide, $0.5 \%$ TBE buffer, $0.07 \%$ APS, and $0.035 \%$ TEMED. The gel was run at constant $120 \mathrm{~V}$ for approximately $3 \mathrm{~h}$ in 1 ? TBE buffer. The gel was silverstained according to Lis procedure [27], and was then documented using a scanner. The genotype was determined by analysis of the bands pattern, dependent on the number and the position of bands. 
Table 1 Fifty nine cymbidium accessions used for genetic analysis

\begin{tabular}{|c|c|c|c|}
\hline Accession & Name & Group $^{a}$ & Species \\
\hline 1 & Tiegusu & 4 & C. ensifolium \\
\hline 2 & Qingshanyuquan & 4 & C. ensifolium \\
\hline 3 & Jinsimawei & 4 & C. ensifolium \\
\hline 4 & Jinhe & 4 & C. ensifolium \\
\hline 5 & Yinsimawei & 4 & C. ensifolium \\
\hline 6 & Dayibai & 4 & C. ensifolium \\
\hline 7 & Dahongzhusha & 2 & C. ensifolium \\
\hline 8 & Qiuhong & 4 & C. ensifolium \\
\hline 9 & Baodao & 4 & C. ensifolium \\
\hline 10 & Jinhe & 2 & C. ensifolium \\
\hline 11 & Tianhe & 4 & C. ensifolium \\
\hline 12 & Shisantaibao & 4 & C. ensifolium \\
\hline 13 & TuerA & 2 & C. lancifolium \\
\hline 14 & TuerB & 2 & C. lancifolium \\
\hline 17 & DuohualanA & 5 & C. floribundum \\
\hline 18 & GuoxianglanA & 2 & C. suavissimum \\
\hline 19 & ShayelanA & 1 & C. cyperifolium \\
\hline 20 & ShayelanB & 1 & C. cyperifolium \\
\hline 21 & ShayelanC & 1 & C. cyperifolium \\
\hline 22 & ShayelanD & 1 & C. cyperifolium \\
\hline 23 & QiubeidonghuiA & 2 & C. qiubeiense \\
\hline 24 & ShayelanE & 1 & C. cyperifolium \\
\hline 25 & LvlanA & 1 & C. faberi \\
\hline 26 & GuoxianglanB & 5 & C. suavissimum \\
\hline 27 & IvlanB & 1 & C. faberi \\
\hline 28 & DuohualanB & 5 & C. floribundum \\
\hline 29 & Yuhudie & 2 & C. goeringii \\
\hline 30 & Yinhe & 5 & C. goeringii \\
\hline 31 & Silan & 2 & C. goeringii \\
\hline 32 & Hexingmei & 5 & C. goeringii \\
\hline 33 & Dasongmei & 2 & C. goeringii \\
\hline 34 & Yipin & 2 & C. goeringii \\
\hline 35 & Huangmei & 2 & C. goeringii \\
\hline 36 & Puchunhong & 2 & C. goeringii \\
\hline 37 & Chunjiansuxin & 2 & C. goeringii \\
\hline 38 & Hongmeigui & 2 & C. goeringii \\
\hline 39 & Wenyi & 2 & C. goeringii \\
\hline 40 & Jiuxianmudan & 2 & C. goeringii \\
\hline 41 & Dayipin & 3 & C. faberi \\
\hline 42 & Ruyisu & 2 & C. faberi \\
\hline 43 & Jiepeimei & 3 & C. faberi \\
\hline 44 & Xinshanghaimei & 3 & C. faberi \\
\hline 45 & Laoranzi & 3 & C. faberi \\
\hline
\end{tabular}

Table 1 Fifty nine cymbidium accessions used for genetic analysis (Continued)

\begin{tabular}{llll}
\hline 46 & Xiashanjiujielan & 3 & C. faberi \\
47 & Guifei & 3 & C. faberi \\
48 & Mingyue & 3 & C. faberi \\
49 & Xiyang (Qingxiang) & 3 & C. faberi \\
50 & Yuchan & 3 & C. faberi \\
51 & QiubeidonghuiB & 2 & C. qiubeiense \\
52 & DuohualanC & 5 & C. floribundum \\
53 & DuohualanD & 5 & C. floribundum \\
54 & QiubeidonghuiC & 2 & C. qiubeiense \\
57 & Wuzicui & 2 & C. sinense \\
58 & Jinhuashan & 2 & C. sinense \\
59 & Rixiang & 2 & C. sinense \\
60 & Qihei & 2 & C. sinense \\
61 & Damo & 2 & C. sinense \\
62 & Hongmeiren & 2 & C. sinense \\
63 & Baimo & 2 & C. sinense \\
\hline
\end{tabular}

${ }^{\mathrm{a} F i v e ~ g r o u p s ~ i n d i c a t e d ~ b y ~ p o p u l a t i o n ~ s t r u c t u r e ~ a n a l y s i s . ~}$

\section{Statistical analysis}

Genetic distance was calculated using Neis distance [28]. Phylogenetic reconstruction was based on the unweighted pair-group method that utilizes the arithmetic average (UPGMA) method implemented in PowerMarker version 2.7 [29]. The tree that was used to visualize the phylogenetic distribution of accessions and ancestry groups was constructed using MEGA version 4 [30]. A model-based program structure [31] was used to infer population structure with 5,000 burn-in and run length. The model allowed for admixture and correlated allele frequencies. The number of groups (K) was set from 1 to 10, each with 10 independent runs. The most probable structure number (K) was determined through log probability [32]. Principal component analysis (PCA), which summarizes the major patterns of variation in a multi-locus data set, was performed using NTSYSpc version $2.11 \mathrm{~V}$ [33]. Two principal components were used to represent the dispersion of the collection accessions graphically [34]. PowerMarker was used to calculate the average number of marker alleles and the polymorphism information content (PIC) values. Fixation index $(F s t)$, which indicates the differentiation among genetic groups, was calculated using an Analysis of Molecular Variance (AMOVA) approach in Arlequin V2.000 [35].

\section{Results}

\section{Genic-SSR search and primer design}

In C. ensifolium transcriptome, 98,819,349 reads, (9.52 Gb), were obtained after removal of adaptor sequences, ambiguous reads, and low-quality reads $(\mathrm{Q}$-value $<25)$. These reads 
were used for the subsequent assembly, and then resulted in 101,423 unigenes $(139,385,689$ residues). The length of unigenes averaged $1,374 \mathrm{bp}$ and ranged from $351 \mathrm{bp}$ to $17,260 \mathrm{bp}$. The data were uploaded to the NCBI (http:// orchidbase.itps.ncku.edu.tw/est/home2012.aspx) for public use (Accession: SRA098864).

In the present study, 7,936 genic-SSRs were identified, with one SSR locus for every $17.56 \mathrm{~kb}(\mathrm{~kb} / \mathrm{SSR})$. Estimated locations (coding, 5'UTR or 3'UTR) were obtained for 5,524 genic-SSRs. Sequence information could not be determined for the remaining 2,412 genic-SSR regions, because the locations were extended over both estimated coding and non-coding regions. Given such high numbers of SSR, we analyzed the sequence data to isolate high quality SSR loci for further testing. An important factor considered was the locations of SSRs relative to ORFs. SSRs within UTR are exposed to lower selective pressure than those in coding regions and have a higher likelihood of being polymorphic [36]. Another two factors are the length of the motif and the number of the repeat motif, which are often associated with polymorphism [37]. Thus, SSRs within UTR, with short motifs and high repeat number would be the best marker candidates. Herein, we selected 80 genic-SSRs and designed primers based on their motifs, sizes and locations.

\section{Genic-SSRs profile}

All primer sets were initially tested among 12 C. ensiflolium accessions, and then were cross-tested among other 47 Cymbidium accessions (Table 1). Of the 80 genic-SSR primers, 62 amplified within C. ensifolium accessions successfully, and 55 showed polymorphism when crosstested among all 9 cymbidium species (Additional file 1: Figure S1). These accessions belonged to 9 cymbidium species i.e. C. ensifolium, C. lancifolium, C. suavissimum, C. cyperifolium, C. qiubeiense, C. floribundum, C. goeringii, C. faberi and C. sinense. Among the 55 polymorphic markers, the PIC averaged 0.407, ranging from 0.033 (for both SSR29 and SSR31) to 0.863 (for SSR73). Similarly, allele number averaged 5.75, ranging from 2 (for SSR06, SSR24, SSR29, SSR31, SSR46, SSR55, SSR71, SSR75 and SSR79) to 16 (for SSR73) (Table 2). These results suggested that genic-SSR markers had a broad applicability within Cymbidium genus.

\section{Genetic diversity and population structure}

These genic-SSRs revealed genetic variation among accessions. The genetic distance among accessions ranged from 0.016 to 0.618 , with an average of 0.391 . The model-based clustering method revealed five groups (Figure 1A and B). Group 2 had the most accessions (26), with the highest mean genetic distance (MGD) of 0.431 among these accessions; Group 4 had 10, with an average distance of 0.236; Group 5 had 7, with MGD of 0.332; Group 1 and Group5 both had 7 accessions, with MGD of 0.155 and 0.332 , respectively; Group 3 had 9, with MGD of 0.213. Genetic distance among five groups was from 0.340 (between group 1 and group 5) to 0.176 (between group 2 and group 4, with average of 0.248) (Table 3).

The five groups revealed by the model-based clustering analysis consisted of different species. Three groups comprised more than one species, whereas the other two only comprised one species. Group 1 included two species i.e. C. cyperifolium and C. goeringii; Group 2 included C.ensifolium, C. lancifolium, C. suavissimum, C. qiubeiense, C. goeringii, C. faberi, and C. sinense; Group 5 included C. floribundum, C. suavissimum and C. goeringii. Goup 3 and Group 4 included only C. faberi and C.ensifolium, respectively (Figure 2).

The first two components in PCA $(47.87 \%$ and $21.59 \%$ of total variation, respectively) discriminated the five groups at a certain level. Basically, accessions in group 1 and group 3 stayed alone, whereas group 2 overlapped with group 4 and group 5 (Figure 1C). In the phylogenetic tree, group 2 and group 4 were genetically close, while group 5 was relatively distant from the other groups (Figure 1A). In addition, a few accessions in group 2 had admixture ancestry from group 3 and group 4, while accessions in group 3 and group 1 had less admixture ancestry (Figure 1B). AMOVA results showed that $25.34 \%$ of the total variation was among groups, while $74.66 \%$ of the variation was within groups. The $F_{S T}$ was 0.25 , as indicated by the AMOVA approach.

\section{Genic-SSR annotation}

Annotations of these unigenes provide biological information for 62 genic-SSRs, such as KOG clusters, GO, and KEGG pathway information. Distinct gene sequences were first searched using BLASTX against the $\mathrm{Nr}$ database. The results showed that 53 unigenes had hits that exceeded the E-value threshold. In the present study, 39 unigenes were categorized into 25 GO terms in three GO ontologies (Figure 3A). Two groups membrane and nucleus, one group binding, and one group cellular process comprised the most representative genes found in cellular components, molecular function, and biological processes, respectively. Out of 53 hits in the $\mathrm{Nr}$ databases, 18 sequences were classified into 9 KOG categories (Figure 3B). Among the 9 KOG categories, General function prediction only and Posttranslational modification, protein turnover, chaperones were the two largest groups. When referenced to rice (Oryza sativa), 15 unigenes were found to be involved in 14 pathways (Figure 3C). The most highly representative one was metabolic pathways, where unigenes shared similarity with 18 rice sequences. 
Table 2 List of the 62 C. ensifolium genic-SSR primers including their unigenes annotation

\begin{tabular}{|c|c|c|c|c|c|c|c|c|c|c|}
\hline Name & $\begin{array}{l}\text { Product } \\
\text { size (bp) }\end{array}$ & SSR & $\begin{array}{l}\text { SSR } \\
\text { location }\end{array}$ & Primer & Homologs in non-redundant database (accession in Genebank) & $\begin{array}{l}\text { GO } \\
\text { annotation }\end{array}$ & $\begin{array}{l}\text { KOG } \\
\text { annotation }\end{array}$ & $\begin{array}{l}\text { KEGG } \\
\text { annotation }\end{array}$ & $\begin{array}{l}\text { Allele } \\
\text { number }\end{array}$ & PIC \\
\hline \multirow[t]{2}{*}{ SSR01 } & $400-500$ & $(\mathrm{AC}) 8$ & utr5 & F: AACGCCATGTCCAATACCC & PREDICTED: probable transcription factor KAN2-like (XP_002278005.2) & GO: 0003677 & KOG1601 & NULL & 5 & 0.552 \\
\hline & & & & R: GGAGGGCTTATTTGCAGCG & & & & & & \\
\hline \multirow[t]{2}{*}{ SSR02 } & $300-400$ & $(\mathrm{AC}) 8$ & utr5 & F: CTCCTTCAAGCTTCTGCCC & $\begin{array}{l}\text { PREDICTED: histone-lysine N-methyltransferase, H3 lysine-9, H3 } \\
\text { lysine-27, H4 lysine-20 and cytosine specific SUVH2 (XP_002282386.1) }\end{array}$ & GO: 0042393 & NULL & NULL & NA & NA \\
\hline & & & & R: GACCGCAGCGTTAATGACC & & & & & & \\
\hline \multirow[t]{2}{*}{ SSR03 } & $400-500$ & $(\mathrm{AC}) 8$ & utr3 & F: CTCGGTTCATTTGCAGCCC & $\begin{array}{l}\text { PREDICTED: mitochondrial import receptor subunit TOM20 } \\
\text { (XP_002269795.1) }\end{array}$ & GO: 0045040 & NULL & NULL & 7 & 0.690 \\
\hline & & & & R: GGGTGGGTATGGCGAAATC & & & & & & \\
\hline \multirow[t]{2}{*}{ SSRO4 } & $400-500$ & $(\mathrm{AC}) 8$ & utr3 & F: AGAATCTGCCAACCCTTGATAC & NULL & NULL & NULL & NULL & 6 & 0.657 \\
\hline & & & & R: GCAGATGCCAGTTAGAATGGG & & & & & & \\
\hline \multirow[t]{2}{*}{ SSR05 } & 1000 & $(\mathrm{AC}) 8$ & utr3 & F: AGAACTGCAGGTGTGAAGC & PREDICTED: protein CbbY, chromosomal-like isoform 1 (XP_003574671.1) & GO: 0016787 & NULL & NULL & 3 & 0.125 \\
\hline & & & & R: GGCTTGAAGTGGCGATAACC & & & & & & \\
\hline \multirow[t]{2}{*}{ SSR06 } & 600 & (AC)9 & utr3 & F: GCGTCTGCTGAAACGATGG & Putative steroid 22-alpha-hydroxylase (AAN60994.1) & GO: 0016020 & KOG0157 & K09587 & 2 & 0.063 \\
\hline & & & & R: AAACAGCGCCTGTCATTCC & & & & & & \\
\hline \multirow[t]{2}{*}{ SSR07 } & $300-400$ & $(\mathrm{AC}) 9$ & utr3 & F: ACGCTGCATCCCATTTCAC & PREDICTED: uncharacterized protein LOC100243361 (XP_002276849.2) & GO: 0008987 & NULL & K03517 & 4 & 0.180 \\
\hline & & & & R: CAGTCTGTTGAGGAAGCCG & & & & & & \\
\hline \multirow[t]{2}{*}{ SSR08 } & $100-200$ & $(\mathrm{AC}) 10$ & utr3 & F: TGCTGGAATACATGCGAGAC & Predicted protein (XP_002298559.1) & GO: 0023014 & KOG0610 & & 14 & 0.753 \\
\hline & & & & R: GTTTGCCGAAGCCAGTGC & & & & & & \\
\hline \multirow[t]{2}{*}{ SSR11 } & 600 & $(\mathrm{AG}) 10$ & utr3 & F: AACTGACAAGCATCTGCAAG & Uncharacterized protein LOC100273319 precursor (NP_001141232.1) & GO: 0005774 & NULL & NULL & 6 & 0.477 \\
\hline & & & & R: CTGCTGCATTGGCCTTACC & & & & & & \\
\hline \multirow[t]{2}{*}{ SSR12 } & 300 & $(A G) 11$ & utr5 & F: TCAGCCGAGGTTAGTATACGG & $\begin{array}{l}\text { PREDICTED: phosphatidylinositol-4-phosphate 5-kinase 9-like } \\
\text { (XP_002265706.1) }\end{array}$ & GO: 0016020 & KOG0229 & K00889 & NA & NA \\
\hline & & & & R: CTTGCCATCTCAGCAGTCG & & & & & & \\
\hline \multirow[t]{2}{*}{ SSR13 } & $400-500$ & $(\mathrm{AG}) 11$ & utr5 & F: GCTGCTGCTTGGTGGAAAC & Predicted protein (XP_002317724.1) & GO: 0005488 & NULL & NULL & 6 & 0.343 \\
\hline & & & & R: GCGCTCGTTGTATGGCTTG & & & & & & \\
\hline \multirow[t]{2}{*}{ SSR14 } & 300 & $(A G) 11$ & utr5 & F: CACAGCAGCTCACAATCCTG & Unnamed protein product (CBI20568.3) & GO: 0006099 & KOG1257 & K00029 & 8 & 0.467 \\
\hline & & & & R: TACAGCCCTGTTTACCGCC & & & & & & \\
\hline \multirow[t]{2}{*}{ SSR15 } & $100-200$ & $(\mathrm{AG}) 11$ & utr3 & F: CCTTCTCTCCGCGTACCAG & PREDICTED: uncharacterized protein LOC100825549 (XP_003558805.1) & GO: 0005783 & NULL & NULL & 4 & 0.339 \\
\hline & & & & R: CTTCGGTTGGCGTTTAGGG & & & & & & \\
\hline \multirow[t]{2}{*}{ SSR16 } & $300-400$ & $(\mathrm{AG}) 11$ & utr5 & F: GCCCACAGCAATCCATCTG & PE repeat family protein (XP_003014087.1) & NULL & NULL & NULL & 7 & 0.348 \\
\hline & & & & R: GCAGTCGAAGAAACCGTGG & & & & & & \\
\hline \multirow[t]{2}{*}{ SSR17 } & 400 & $(A G) 11$ & utr5 & F: GGATCACCAACAGCATGGG & Transcription factor (ADG57844.1) & GO: 0003677 & NULL & K09060 & 4 & 0.417 \\
\hline & & & & R: TCCACCAAGAGCAAGGATG & & & & & & \\
\hline
\end{tabular}


Table 2 List of the 62 C. ensifolium genic-SSR primers including their unigenes annotation (Continued)

\begin{tabular}{|c|c|c|c|c|c|c|c|c|c|c|}
\hline \multirow[t]{2}{*}{ SSR18 } & 300 & $(\mathrm{AG}) 11$ & utr5 & F: TGAAACGGTTGGCTCTAGTTC & Conserved hypothetical protein (XP_002527260.1) & NULL & NULL & NULL & 13 & 0.519 \\
\hline & & & & R: AGCAAGCACTGACCTGAAAC & & & & & & \\
\hline \multirow[t]{2}{*}{ SSR21 } & $300-500$ & $(\mathrm{GTT}) 8$ & utr3 & F: TGGGCGACAGATCGAGTTC & Hypothetical protein OsJ_08996 (EAZ25197.1) & NULL & NULL & NULL & 15 & 0.794 \\
\hline & & & & R: ACATGGACCACAGCATTCC & & & & & & \\
\hline \multirow[t]{2}{*}{ SSR22 } & $200-300$ & $(\mathrm{GTT}) 9$ & utr3 & F: TATGCGTCTCTCCCAACCG & 14-3-3-like protein B-like(ACQ45020.1) & GO: 0019904 & KOG0841 & K06630 & 10 & 0.572 \\
\hline & & & & R: AAGCTAGTGGCCTTTGGTG & & & & & & \\
\hline \multirow[t]{2}{*}{ SSR23 } & $100-200$ & (GT) 10 & utr3 & F: CGGCGATCGATTATGAGCC & PREDICTED: beta-amylase 1, chloroplastic isoform 1 (XP_002285569.1) & GO: 0005634 & NULL & K01177 & NA & NA \\
\hline & & & & R: CGATACTCCTCAATGTCGTGG & & & & & & \\
\hline \multirow[t]{2}{*}{ SSR24 } & $200-300$ & (GT)11 & utr5 & F: TCGGTAACCTGTTGCAAGG & $\begin{array}{l}\text { PREDICTED: flavin-containing monooxygenase YUCCA6-like } \\
\text { (XP_003550114.1) }\end{array}$ & GO: 0050661 & NULL & K11816 & 2 & 0.063 \\
\hline & & & & R: ACCTGTGAAGCTACCAGAC & & & & & & \\
\hline \multirow[t]{2}{*}{ SSR25 } & $100-250$ & $(\mathrm{GT}) 11$ & utr3 & F: GAATCTCTCGCACCCGAAG & $\begin{array}{l}\text { Aspartyl/glutamyl-tRNA(Asn/Gln) amidotransferase subunit B, putative } \\
\text { (XP_002528338.1) }\end{array}$ & GO: 0006536 & NULL & K02434 & NA & NA \\
\hline & & & & R: TGGACAACATCAAGTGACGC & & & & & & \\
\hline \multirow[t]{2}{*}{ SSR26 } & $100-250$ & $(\mathrm{AAG}) 7$ & utr3 & F: GCTTTATGCGACATCTGCG & Unnamed protein product (CBI25980.3) & GO: 0005634 & KOG1901 & NULL & 11 & 0.638 \\
\hline & & & & R: CGTCGGTTCCATGCACATC & & & & & & \\
\hline \multirow[t]{2}{*}{ SSR27 } & $500-600$ & $(\mathrm{AGC}) 5$ & utr3 & F: CTGCCTTCACAGCTAATGCC & Os04g0512400 (NP_001053298.1) & GO: 0046872 & NULL & NULL & 3 & 0.313 \\
\hline & & & & R: GCATGCTTGGACGCTGAAC & & & & & & \\
\hline \multirow[t]{2}{*}{ SSR29 } & $200-300$ & $(A G C) 6$ & utr3 & F: AGCAAACGGCAAGTCATGG & RING finger protein 113A, putative (XP_002522169.1) & GO: 0016020 & NULL & K13127 & 2 & 0.033 \\
\hline & & & & R: ATTCGACTACCAGCCGGAC & & & & & & \\
\hline \multirow[t]{2}{*}{ SSR30 } & 200-300 & $(\mathrm{AGG}) 5$ & utr3 & F: AAACGAAGGGCTGGAAGTC & NULL & NULL & NULL & NULL & 9 & 0.486 \\
\hline & & & & R: TTTGACATCGGGAAGTGGC & & & & & & \\
\hline \multirow[t]{2}{*}{ SSR31 } & $100-200$ & $(\mathrm{AGG}) 5$ & utr5 & F: GGGATGCATAGACCTTTCGC & Protein MSF1, putative (XP_002535293.1) & GO: 0005739 & KOG3336 & NULL & 2 & 0.033 \\
\hline & & & & R: CAGGTTCAACGGCATCGTG & & & & & & \\
\hline \multirow[t]{2}{*}{ SSR32 } & $1000-1100$ & $(A G G) 5$ & utr3 & F: CTCCGGCCTCTGGTTACTC & PREDICTED: HVA22-like protein j (XP_002281038.1) & NULL & KOG1726 & NULL & 7 & 0.601 \\
\hline & & & & R: AGTGATGAGGCTTGGACCG & & & & & & \\
\hline \multirow[t]{2}{*}{ SSR34 } & $700-900$ & $(A G G) 6$ & utr5 & F: GAGAGGGAATTGCAGTGGC & Hypothetical protein (BAl68347.1) & NULL & NULL & NULL & 6 & 0.696 \\
\hline & & & & R: ACCGAGCTAGCACTTCATC & & & & & & \\
\hline \multirow[t]{2}{*}{ SSR35 } & $700-900$ & $($ ATC) 5 & utr5 & F: AGAGTGATTGTCCAGCTCCG & PREDICTED: diacylglycerol kinase-like (XP_003534537.1) & GO: 0009395 & NULL & K07029 & 4 & 0.475 \\
\hline & & & & R: TGCCTCTCTGGTGATGTCC & & & & & & \\
\hline \multirow[t]{2}{*}{ SSR36 } & $400-500$ & (ATC)5 & utr3 & F: AGTATTGGACCCTCCAGGC & NULL & NULL & NULL & NULL & 5 & 0.536 \\
\hline & & & & R: AGAGGATCATGGTGTTAGGC & & & & & & \\
\hline \multirow[t]{2}{*}{ SSR37 } & $200-300$ & $(\mathrm{ATC}) 5$ & utr5 & F: GGCCTAGCCAGCCCTTC & NULL & NULL & NULL & NULL & 3 & 0.205 \\
\hline & & & & R: ATTTGGATCGCACAAGCGG & & & & & & \\
\hline
\end{tabular}


Table 2 List of the 62 C. ensifolium genic-SSR primers including their unigenes annotation (Continued)

\begin{tabular}{|c|c|c|c|c|c|c|c|c|c|c|}
\hline \multirow[t]{2}{*}{ SSR38 } & 200-300 & (ATC)6 & utr3 & F: TAGCCCATGCCAGTGTTCC & LOC100285373 (NP_001151738.1) & GO: 0007165 & NULL & NULL & 3 & 0.149 \\
\hline & & & & R: AACTGCCACAAGAGAAGGC & & & & & & \\
\hline \multirow[t]{2}{*}{ SSR39 } & $1000-1100$ & (ATC)6 & utr3 & F: ACAGACTGCCACCTGTTCC & unnamed protein product (CB|38283.3) & GO: 0008234 & KOG1870 & K11835 & 5 & 0.401 \\
\hline & & & & R: GCCTGCCTTTGCTCCTTG & & & & & & \\
\hline \multirow[t]{2}{*}{ SSR40 } & 400 & (ATC)6 & utr5 & F: ACAAGCATCATCCCAAATTCC & $\begin{array}{l}\text { PREDICTED: probably inactive leucine-rich repeat receptor-like protein } \\
\text { kinase At2g25790-like (XP_002267653.1) }\end{array}$ & GO: 0007165 & NULL & NULL & NA & NA \\
\hline & & & & R: GCAGAAACTGGAGCTTGCC & & & & & & \\
\hline \multirow[t]{2}{*}{ SSR42 } & 200-300 & $(\mathrm{CCG}) 5$ & utr5 & F: GACGACATATCGCGTTCGG & unnamed protein product (CBI18667.3) & GO: 0003779 & KOG0160 & NULL & 6 & 0.564 \\
\hline & & & & R: CTCAGCCACACCCAAGAGG & & & & & & \\
\hline \multirow[t]{2}{*}{ SSR43 } & 500 & (CCG) 5 & utr5 & F: GGAGCTGCATACGCAAGTG & glycinebetaine/proline transporter (BAJ07206.1) & GO: 0015193 & NULL & NULL & 7 & 0.572 \\
\hline & & & & R: AGCTTCTCACTGCCTCCAG & & & & & & \\
\hline \multirow[t]{2}{*}{ SSR44 } & $300-400$ & $(\mathrm{CCG}) 5$ & utr5 & F: CGTCGACTCCTCGAGATCC & predicted protein (BAJ93650.1) & GO: 0046872 & NULL & NULL & NA & NA \\
\hline & & & & R: GCGTTAGCAGCAGTCTTGG & & & & & & \\
\hline \multirow[t]{2}{*}{ SSR45 } & $400-500$ & $(\mathrm{CCG}) 5$ & utr5 & F: GCCTTACACATCCCTTCCAAC & unnamed protein product (CBI33381.3) & GO: 0005515 & KOG0550 & NULL & 5 & 0.338 \\
\hline & & & & R: TGCCTGCTGATAGTTTGCC & & & & & & \\
\hline \multirow[t]{2}{*}{ SSR46 } & 200-300 & (CCG)6 & utr5 & F: CCTTCGTGGACTCAACAGC & hypothetical protein SORBIDRAFT_01g031510 (XP_002465065.1) & NULL & NULL & NULL & 2 & 0.063 \\
\hline & & & & R: TCTCGTGCAGGAATCGGTC & & & & & & \\
\hline \multirow[t]{2}{*}{ SSR47 } & $400-500$ & (CCG)6 & utr3 & F: GCAGGTGTCCTCATCGGAG & CONSTANS-like protein (ADN97077.1) & GO: 0005622 & KOG1601 & NULL & NA & NA \\
\hline & & & & R: CTCCGGCTAACTCCATCCC & & & & & & \\
\hline \multirow[t]{2}{*}{ SSR49 } & 300 & $(\mathrm{CCG}) 7$ & utr3 & F: AGAGGGCCACCTGCTTTC & predicted protein (XP_002312577.1) & NULL & KOG1863 & NULL & 6 & 0.549 \\
\hline & & & & R: GCCAATTGCCAGATGGACG & & & & & & \\
\hline \multirow[t]{2}{*}{ SSR52 } & $400-500$ & $(\mathrm{CCT}) 4$ & utr5 & F: AAGAGGCACTGCAAGACCC & hypothetical protein SORBIDRAFT_01g031070 (XP_002465040.1) & NULL & NULL & NULL & 8 & 0.378 \\
\hline & & & & R: CGTTCCAGCAACCCATAGC & & & & & & \\
\hline \multirow[t]{2}{*}{ SSR53 } & $100-200$ & $(\mathrm{CCT}) 4$ & utr5 & F: GCTGAAGGTTCCGGTCCTC & PREDICTED: uncharacterized protein LOC100830480 (XP_003580351.1) & NULL & NULL & NULL & 8 & 0.700 \\
\hline & & & & R: TCCGCCTCTITAAGCCGAC & & & & & & \\
\hline \multirow[t]{2}{*}{ SSR54 } & 200-300 & (CCT) 4 & utr5 & F: ATCTTCCCTCCACATCGGC & hypothetical protein MTR_1g083540 (XP_003591171.1) & GO: 0005886 & NULL & NULL & 5 & 0.422 \\
\hline & & & & R: TGGAGAAGAGTCGACCAGC & & & & & & \\
\hline \multirow[t]{2}{*}{ SSR55 } & 200-300 & (CCT) 4 & utr5 & F: TGGAATGGTTCTAGGGCTTC & hypothetical protein (CCA65980.1) & NULL & NULL & NULL & 2 & 0.323 \\
\hline & & & & R: CCACTGGTACCCTCCTTGG & & & & & & \\
\hline \multirow[t]{2}{*}{ SSR56 } & $900-1000$ & $(\mathrm{CCT}) 5$ & utr5 & F: TGCTTCATTGTTGGAGGCG & predicted protein (XP_002324427.1) & GO: 0008643 & KOG0254 & NULL & 5 & 0.315 \\
\hline & & & & R: AGTGGACGGAGAGTCAAGC & & & & & & \\
\hline \multirow[t]{2}{*}{ SSR59 } & 200-300 & $(\mathrm{CGG}) 5$ & utr3 & F: GTTTCCAACGGTCAGCTCG & $\begin{array}{l}\text { leucine-rich repeat transmembrane protein kinase family protein } \\
\text { (NP_177007.1) }\end{array}$ & GO: 0005524 & NULL & NULL & 3 & 0.432 \\
\hline & & & & R: GTGATGTGGTAGCATCGCC & & & & & & \\
\hline
\end{tabular}


Table 2 List of the 62 C. ensifolium genic-SSR primers including their unigenes annotation (Continued)

\begin{tabular}{|c|c|c|c|c|c|c|c|c|c|c|}
\hline \multirow[t]{2}{*}{ SSR60 } & 200-300 & (CGG)5 & utr5 & F: TACGGTTTCGACCAGCCTC & Unnamed protein product (CBI41056.3) & GO: 0005634 & KOG0265 & K10143 & 4 & 0.203 \\
\hline & & & & R: CCATGCAGATCGGGCAAAG & & & & & & \\
\hline \multirow[t]{2}{*}{ SSR62 } & $300-400$ & $(\mathrm{CGG}) 6$ & utr5 & F: GGTGGGTTAGACCAGCTCC & Hypothetical protein Osl_29809 (EAZ07555.1) & GO: 0005634 & NULL & NULL & 6 & 0.570 \\
\hline & & & & R: TCCTCAAGGCAAAGCTCCC & & & & & & \\
\hline \multirow[t]{2}{*}{ SSR63 } & $100-200$ & (CGG)6 & utr5 & F: CTTCCTCCACCTGGATCGC & Uncharacterized protein LOC100277474(NP_001144494.1) & GO: 0008270 & NULL & NULL & 4 & 0.308 \\
\hline & & & & R: CTGCCGATCAATCCGAGAC & & & & & & \\
\hline \multirow[t]{2}{*}{ SSR64 } & $400-500$ & (CGG)6 & utr3 & F: CGCTCAAAGAGATGGCACG & Os01g0226200 (NP_001042462.1) & NULL & NULL & NULL & 11 & 0.627 \\
\hline & & & & R: TAGTACGGCGCTGCTTGAG & & & & & & \\
\hline \multirow[t]{2}{*}{ SSR66 } & $300-400$ & (CGG)7 & utr3 & F: CATCTTCCTTGCCCGATGC & $\begin{array}{l}\text { PREDICTED: pentatricopeptide repeat-containing protein At5g42310, } \\
\text { mitochondrial (XP_002272226.1) }\end{array}$ & NULL & KOG4197 & NULL & 4 & 0.126 \\
\hline & & & & R: CCCGCCAAATTTCGAGACC & & & & & & \\
\hline \multirow[t]{2}{*}{ SSR68 } & $100-200$ & $(\mathrm{GAT}) 5$ & utr3 & F: CCAGATCGAATGGCTACGC & Hypothetical protein VITISV_010525 (CAN79523.1) & GO: 0003723 & NULL & NULL & 4 & 0.211 \\
\hline & & & & R: CAAGGAGCTCGTCGAAGG & & & & & & \\
\hline \multirow[t]{2}{*}{ SSR69 } & 200-300 & $(\mathrm{GAT}) 5$ & utr5 & F: GTTAGGCTAGCAGTGCGG & NULL & NULL & NULL & NULL & 3 & 0.149 \\
\hline & & & & R: TGAGAACGTAGTGAAGTTGCC & & & & & & \\
\hline \multirow[t]{2}{*}{ SSR70 } & 200-300 & (GAT)7 & utr3 & F: CCCAACGCAGAACGATAGC & NULL & NULL & NULL & NULL & 5 & 0.529 \\
\hline & & & & R: CGGTGGCACAAATGGAACG & & & & & & \\
\hline \multirow[t]{2}{*}{ SSR71 } & $400-500$ & (GGT)5 & utr5 & F: GCATCGAAACCACTGTCGC & Hypothetical protein SORBIDRAFT_09g018170 (XP_002439663.1) & NULL & NULL & NULL & 2 & 0.262 \\
\hline & & & & R: CCCTAGCCGGAGTCTCAAC & & & & & & \\
\hline \multirow[t]{2}{*}{ SSR73 } & $100-200$ & (GGT)5 & utr3 & F: GGACACAATGGAGACGAAGG & T4.15 (CCH50976.1) & GO: 0044238 & NULL & NULL & 16 & 0.863 \\
\hline & & & & R: TGCATGAAACCACATGGC & & & & & & \\
\hline \multirow[t]{2}{*}{ SSR75 } & $400-500$ & $(\mathrm{GTT}) 6$ & utr3 & F: GCCTTTGACCATTCCGTGC & Mitogen-activated protein kinase 1 (AEQ28763.1) & GO: 0043622 & KOG0660 & K04371 & 2 & 0.118 \\
\hline & & & & R: GGCCGCCATGAGTAAGAAC & & & & & & \\
\hline \multirow[t]{2}{*}{ SSR76 } & $500-600$ & (GTT)6 & utr5 & F: AGACAGAGAGTCCCTAAAGGC & NULL & NULL & NULL & NULL & 7 & 0.519 \\
\hline & & & & R: CAGGGATGTTAAGTGGGCTG & & & & & & \\
\hline \multirow[t]{2}{*}{ SSR77 } & $300-400$ & (GTT)6 & utr3 & F: TTTGTGGCAGTGGAAAGCG & NULL & NULL & NULL & NULL & 5 & 0.470 \\
\hline & & & & R: TGATACCAATGGCAAGGCG & & & & & & \\
\hline \multirow[t]{2}{*}{ SSR79 } & 200-300 & $(\mathrm{GTT}) 6$ & utr5 & F: AGGATTCATGTAGCCGACCTC & Hypothetical protein OsI_35425 (EEC67831.1) & NULL & NULL & K10728 & 2 & 0.207 \\
\hline & & & & R: TCCCTGAAGGAGGCAAACC & & & & & & \\
\hline \multirow[t]{2}{*}{ SSR80 } & $400-500$ & $(\mathrm{GTT}) 7$ & utr3 & F: GCACCCAGCTTGTTTGAGG & NULL & NULL & NULL & NULL & 8 & 0.626 \\
\hline & & & & R: CCCATACATTACAGGCAAGC & & & & & & \\
\hline
\end{tabular}




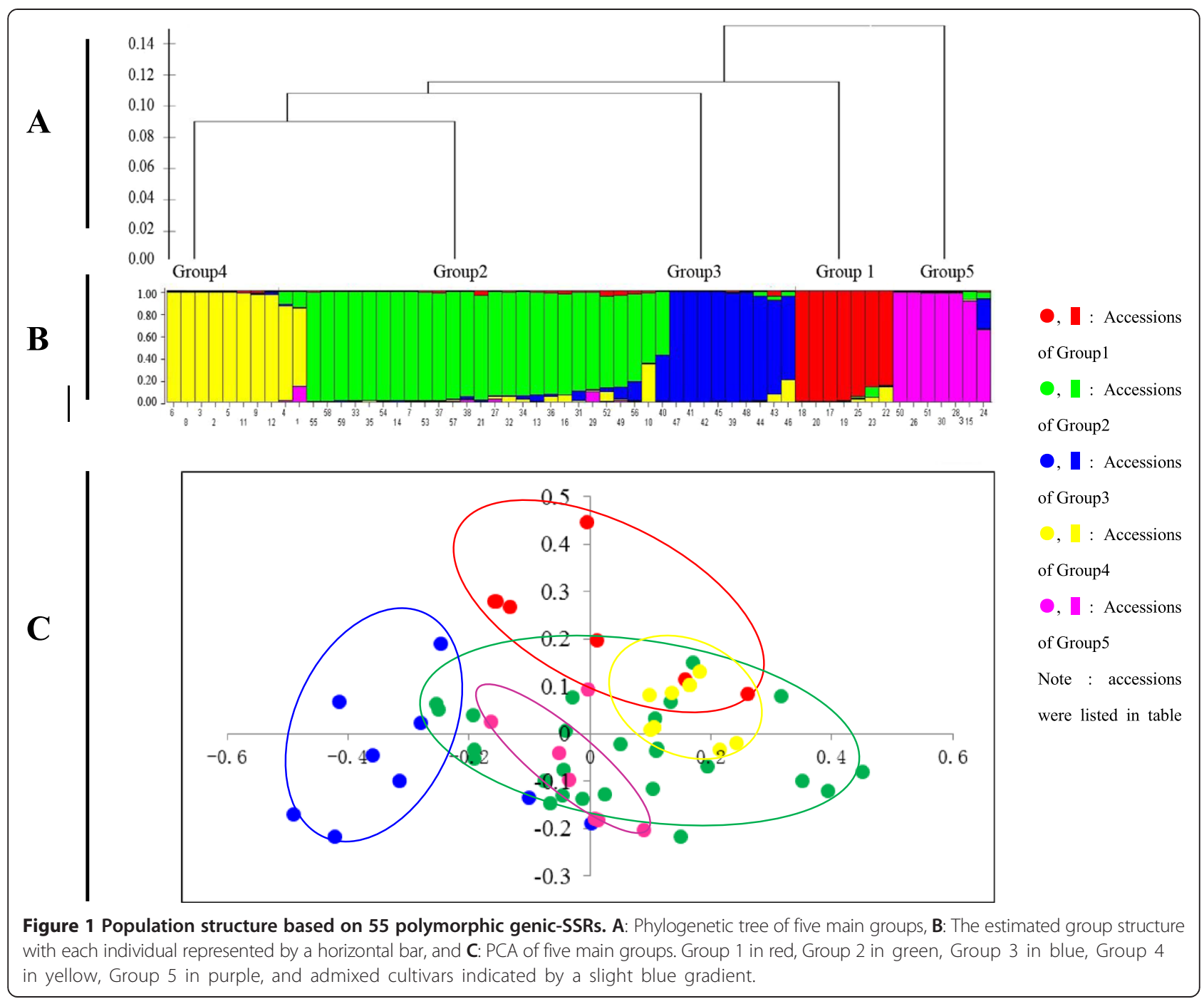

\section{Discussion}

\section{Diversity}

Because genic-SSR markers are derived from transcribed regions of DNA, they are expected to be more conserved and have a higher rate of transferability than anonymous SSR markers [38]. Herein, 55 C. ensifolium polymorphic genic-SSR markers exhibited $100 \%$ transferability across the 59 accessions of the 9 Cymbidium species tested. It is common that genic-SSRs possess a high potential for inter-specific transferability $[39,40]$. Other markers such as RAPDs, ISSR s and non-genic SSRs have also been used with success among $C$. ensifolium and the Cymbidium species reflecting the genetic similarity among many members of the genus $[8,11,15]$.

The conserved nature of the genic-SSRs may limit their polymorphism relative to randomly selected SSRs.

Table 3 Pairwise comparison of Nei s genetic distance among groups and mean of genetic distance within group based on 55 polymorphic genic-SSRs

\begin{tabular}{|c|c|c|c|c|c|c|c|}
\hline Group & No. of accessions & Mean genetic distance (MGD) & Group 1 & Group 2 & Group 3 & Group 4 & Group 5 \\
\hline Group 1 & 7 & 0.155 & 0.000 & & & & \\
\hline Group 2 & 26 & 0.430 & 0.217 & 0.000 & & & \\
\hline Group 3 & 9 & 0.213 & 0.252 & 0.190 & 0.000 & & \\
\hline Group 4 & 10 & 0.236 & 0.212 & 0.176 & 0.234 & 0.000 & \\
\hline Group 5 & 7 & 0.332 & 0.340 & 0.263 & 0.298 & 0.293 & 0.0000 \\
\hline
\end{tabular}




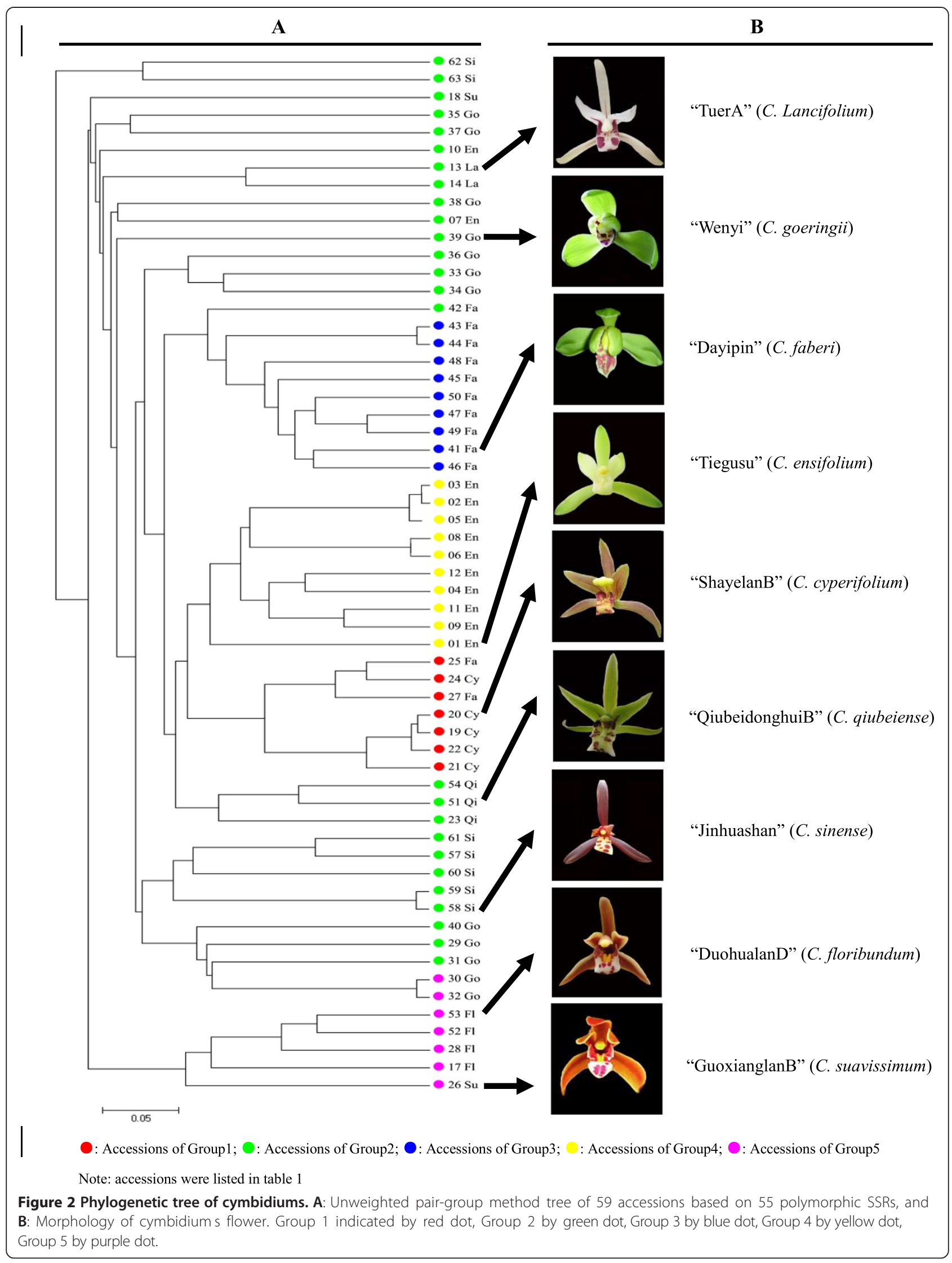




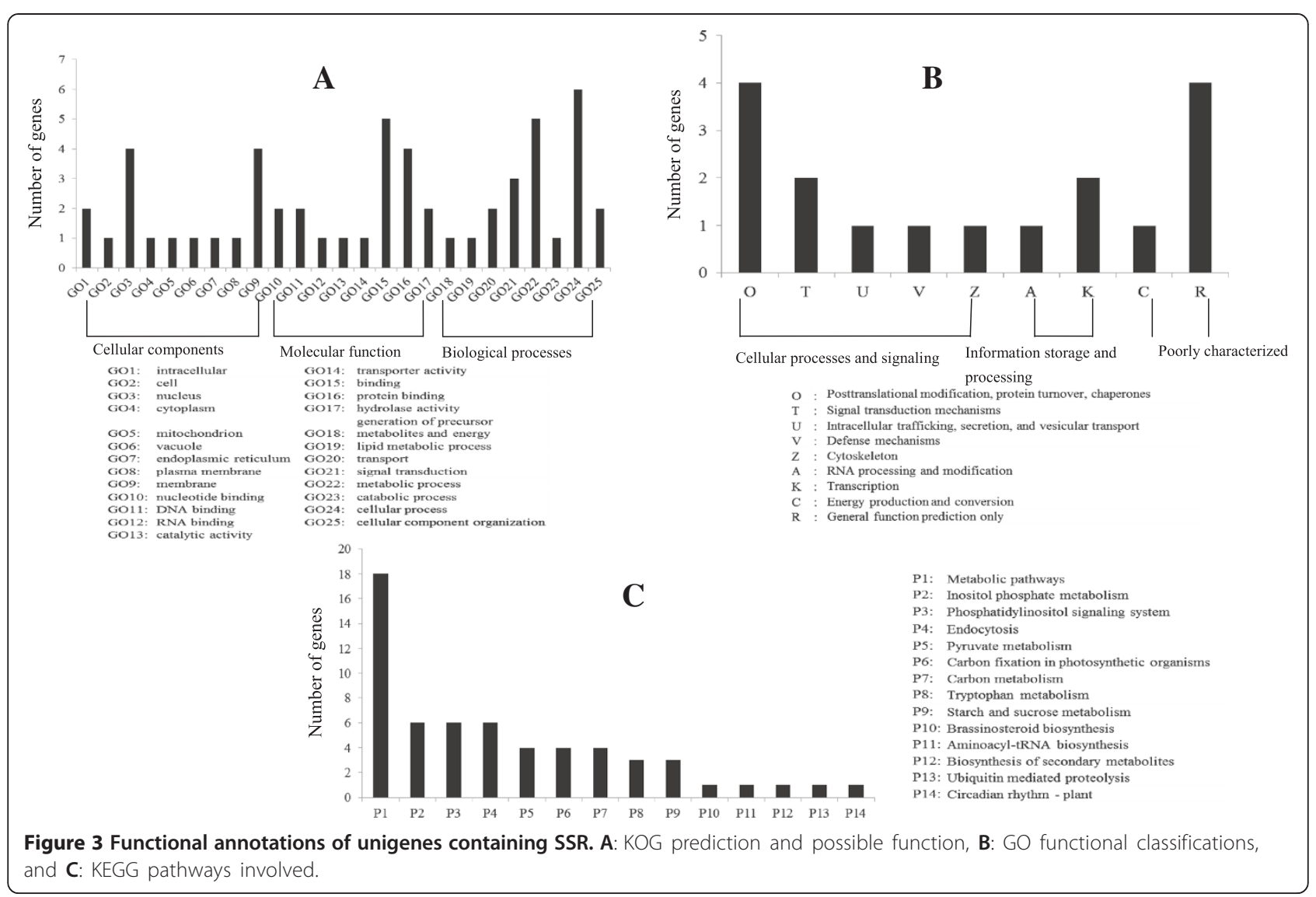

In this study, PIC of genic-SSR markers averaged 0.407, lower than 0.782 [5] and 0.639 [11] of anonymous SSR s tested on Chinese cymbidiums in other studies. The pairwise genetic distance averaged 0.391 among 59 accessions, which is also lower than that from previous studies conducted on Chinese Cymbidiums using other molecular markers [3,8,41-44]. Even though genic-SSRs revealed less variability than SSRs, these markers still reveal sufficient levels of variation for population genetic analysis.

\section{Population structure}

One of the biggest advantages for genic-SSRs is that they allow one to make direct comparisons among taxa without running the risk that locus-specific differences might mask true species-level differences, such as overall levels of genetic diversity, the extent of population structure, and so on. However, the greatest concern with the utilization of genic-SSRs in genetic studies is that selection on these loci might influence the estimation of population genetic parameters. While a recent study by Woodhead et al. [45] revealed that estimates of population differentiation based on genic-SSRs are comparable to those based on both SSRs and AFLPs in ferns, and large-scale comparative analysis suggest that only a very small percentage of all genes has experienced positive selection [46,47], a small fraction of SSRs will be inevitably subject to selection. The view is consistent with the theory that most mutations are neutral, or nearly neutral, [48] or, at least, do not change the function of gene products appreciably [49].

In the population genetic analysis, almost all accessions from the same species clustered together. C. suavissimum and C. floribundum were clustered into one brand, and clearly distinguished from other cymbidiums. Two of them belong to Section Floribundum, and have a distant relationship with other cymbidiums. However, the genetic relationship between C. goeringii and C. sinensis was close, which was congruent with the previous reports $[5,11]$. The close relationship was also found between $C$. ensifolium and C. cyperifolium. In the intersection level, we discovered that two accessions of $C$. faberi were clustered with $C$. cyperifoliumm, and accessions of C. lancifolium and C. ensifolium were scattered among ones of $C$. goeringii. The splitting feature of these clusters might be linked to the non-homologous synapomorphy, even though accessions belonged to different species. The accessions of $C$. goeringii did not always form a separate cluster in the phylogenetic tree or were not grouped together in structure analysis, suggesting that they were polyphyletic. Previous morphologic, cytogenetic, and 
molecular studies have shown that the major lineages of Chinese cymbidiums are ambiguous. C. ensifolium and $C$. sinense are classified in section Jensoa; $C$. faberi and C. goeringii, are classified in section Maxillarianthe; C. faberi, C. kanran, and C. longibracteatum are classified in one group; C. ensifolium, C. goeringii, and C. sinense are categorized into another group [44].

\section{Genic-SSR annotation}

Putative functions were assigned to those unigenes containing SSRs by sequence similarities. These unigenes were involved in a wide range of functions, which indicated that these genic-SSRs were likely important biologically characters. For example, unigene containing SSR47 shares homology with CONSTANS-like protein. In Arabidopsis, the CO (CONSTANS) gene has an important role in the regulation of flowering by photoperiod [50]. Unigene containing SSR43 has homology with a glycinebetaine/proline transporter. The accumulation of glycinebetaine (GB) is one of the adaptive strategies to adverse salt stress conditions [51]. The transporters mediate the uptake of GB and/or proline in many plant species e.g. Arabidopsis thaliana [52], tomato (Solanum lycopersicum) [53], rice (Orazy sativa) [54], barley [55]. Unigene having SSR75, was annotated as mitogen-activated protein kinase (MAPK). MAPK cascades function as key signal transducers that use protein phosphorylation/dephosphorylation cycles to channel information [56]. In the plant, MAPKs have been shown to regulate numerous cellular processes, including biotic stress relief $[57,58]$. Although some unigenes with SSRs had no match to known genes in current gene database, they will likely gain functional annotations as the knowledge of plant genes increases. Compared with anonymous SSRs, genic-SSR markers have a higher probability of being functionally associated with differences in gene expression, which may be in identifying associations between genotype and phenotype. Mapping of genic-SSRs will also provide a map location, in many cases, for genes with known functions.

\section{Conclusion}

In this work, 7,936 genic-SSRs were identified in C. ensifolium transcriptome and their characterizations were further analyzed. A total of 80 genic-SSRs were chosen for validation, and 55 markers successfully yielded polymorphism across 9 Cymbidium species including 59 accessions. The high transferability of genic-SSR will be a powerful resource for molecular taxonomic studies and construction of a reference molecular map of the Cymbidium genome. Since genic-SSR markers belong to generich regions of the genome, some of these can be exploited for use in marker-assisted breeding of Cymbidium. Therefore, the set of genic-SSR markers developed here is a promising genomic resource.

\section{Additional file}

Additional file 1: Figure S1. Polyacrylamide gel electrophoresis profile of SSR62 $a$ and SSR73 b. M: Maker DL2000; 1 63: cymbidium accession listed in Table 1.

\section{Competing interests}

The authors declare that they have no competing interests.

\section{Authors contribution}

$\mathrm{XL}$ and FJ carried out the molecular genetic studies, participated in the sequence alignment and drafted the manuscript. $L J, A J$ and $\mathrm{CH}$ participated in the sequence alignment. $X \mathrm{~L}, \mathrm{FJ}$, and $\mathrm{KL}$ participated in the design of the experiment and performed the statistical analysis. $\mathrm{XL}$, and $\mathrm{XS}$ conceived of the study, and participated in its design and coordination and helped to draft the manuscript. All authors read and approved the final manuscript.

\section{Acknowledgments}

The authors thank Lin Biao for critical review, the lab of Professor Dianxing Wu for materials supply and technical support, and Chongbo Sun for a part of materials supply. This research was supported by the National Basic Research Program funded by the Nature Science Foundation of China (No. 31201648), the Postdoctoral Science Foundation of China (No. 2012 M521203), the Special Postdoctoral Science Foundation of China (No. 2013 T60607), and the Foundation for Selected Postdoctoral project of Zhejiang (Bsh1201032), the Qianjiang talents project (No. 2013R10081), and Scientific and technical innovation promotion project of ZAAS (2012R05Y01E04).

\section{Author details}

'Zhejiang Academy of Agricultural Sciences, Shiqiao Road 139, Hangzhou 310021, Peoples Republic of China. ${ }^{2}$ Hubei University, College of Life Sciences, Wuhan 430062, People s Republic of China. ${ }^{3}$ USDA-ARS, Dale Bumpers National Rice Research Center, Stuttgart, Arkansas 72160, USA. ${ }^{4}$ Agricultural Technology Extension Stations, Shaoxing County Agricultural Bureau, Shaoxing 312000, Peoples Republic of China. ${ }^{5}$ School of Biological Sciences, The University of Hong Kong, Pokfulam Road, Hong Kong, SAR, People s Republic of China. 'State Key Lab of Rice Biology, International Atomic Energy Agency Collaborating Center, Zhejiang University, Hangzhou 310029, Peoples Republic of China.

Received: 14 May 2014 Accepted: 30 October 2014

Published online: 05 December 2014

\section{References}

1. Liu ZJ, Chen SC, Ru ZZ, Chen LJ: Chinese Cymbidium plants. Beijing: Science; 2006.

2. DuPuy D, Cribb PJ: The genus Cymbidium. London: Christopher Helm; 1988.

3. Obara-Okeyo P, Kako S: Genetic diversity and identification of Cymbidium cultivars as measured by random amplified polymorphic DNA (RAPD) markers. Euphytica 1998, 99:95 101.

4. Wang L-m, Wang S-q, Yang Y-f: Germ Plasm Resources and Breeding of Orchids. J Anyang Institute Technol 2005, 2:1 14.

5. Li X, Xiang L, Wang Y, Luo J, Wu C, Sun C, Xie M: Genetic diversity, population structure, pollen morphology and cross-compatibility among Chinese Cymbidiums. Plant Breed 2014, 133:145 152.

6. Escudero A, Iriondo JM, Torres ME: Spatial analysis of genetic diversity as a tool for plant conservation. Biol Conserv 2003, 113:351 365.

7. Ellis JR, Burke JM: EST-SSRs as a resource for population genetic analyses. Heredity 2007, 99:125 132.

8. Choi H, Kim MJ, Lee JS, Ryu KH: Genetic diversity and phylogenetic relationships among and within species of oriental cymbidiums based on RAPD analysis. Sci Hortic 2006, 108:79 85

9. Lu J, Hu X, Liu J, Wang H: Genetic diversity and population structure of 151 Cymbidium sinense cultivars. J Horticulture Forestry 2011, 3:104 114

10. Capesius I: Isolation and characterization of native AT-rich satellite DNA from nuclei of the orchid Cymbidium. FEBS Lett 1976, 68:255 258.

11. Moe KT, Zhao W, Song HS, Kim YH, Chung JW, Cho YI, Park P, Park HS, Chae SC, Park YJ: Development of SSR markers to study diversity in the genus Cymbidium. Biochem Syst Ecol 2010, 38:585 594. 
12. Venkateswarlu M, Raje Urs S, Surendra Nath B, Shashidhar HE, Maheswaran M Veeraiah TM, Sabitha MG: A first genetic linkage map of mulberry (Morus spp.) using RAPD, ISSR, and SSR markers and pseudotestcross mapping strategy. Tree Genet Genomes 2006, 3:15 24

13. Kenis K, Keulemans J: Genetic linkage maps of two apple cultivars (Malus x domestica Borkh.) based on AFLP and mocrosatellite markers. Mol Breed 2005, 15:205 219.

14. RI R, Angiolillo A, Guerrero C, Pellegrini M, Rallo L, Besnard G, Bervill A, Martin A, Baldoni L: A first linkage map of olive (Oleaeuropaea L.) cultivars using RAPD, AFLP, RFLP and SSR markers. Theor Appl Genet 2003, 106:1273 1282

15. Li X, Xiang L, Luo J, Hu B, Tian S, Xie M, Sun C: The strategy of RNA-seq application and development of molecular marker derived from RNA-Seq Chinese J Cell Biol 2013, 5:35.

16. Li X, Cui H, Zhang M: Molecular markers derived from EST: Their development and applications in comparative genomics. Biodiversity Sci 2006, 14:541 547

17. Li X, Luo J, Yan T, Xiang L, Jin F, Qin D, Sun C, Xie M: Deep sequencing-based analysis of the Cymbidium ensifolium floral transcriptome. PLoS One 2013, 8(12):e85480.

18. Grabherr MG, Haas BJ, Yassour M, Levin JZ, Thompson DA, Amit I, Adiconis X, Fan L, Raychowdhury R, Zeng Q, Chen Z, Mauceli E, Hacohen N, Gnirke A, Rhind N, di Palma F, Birren BW, Nusbaum C, Lindblad-Toh K, Friedman N, Regev A: Full-length transcriptome assembly from RNA-Seq data without a reference genome. Nat Biotechnol 2011, 29:644 652.

19. Faircloth BC: Msatcommander: detection of microsatellite repeat arrays and automated, locus-specific primer design. Mol Ecol Resour 2008, 8:92 94

20. Rozen S, Skaletsky H: From methods in molecular Biology: Primer3 on the www for general users and for biologist programmers. In Bioinformatics Methods and Protocols volume 132. Edited by Misener S, Krawetz SA. New Jersey: Humana Press; 2000:365 385.

21. Conesa A, Gotz S, Garcia-Gomez JM, Terol J, Talon M, Robles M: Blast2GO: a universal tool for annotation, visualization and analysis in functional genomics research. Bioinformatics 2005, 21:3674 3676.

22. Kanehisa M, Goto S, Sato Y, Furumichi M, Tanabe M: KEGG for integration and interpretation of large-scale molecular datasets. Nucleic Acids Res 2012, 40:109 114

23. Kanehisa M, Goto S: KEGG: Kyoto Encyclopedia of Genes and Genomes. Nucleic Acids Res 2000, 28:27 30.

24. Koonin EV, Fedorova ND, Jackson JD, Jacobs AR, Krylov DM, Makarova KS, Mazumder R, Mekhedov SL, Nikolskaya AN, Rao BS, Rogozin IB, Smirnov S, Sorokin AV, Sverdlov AV, Vasudevan S, Wolf YI, Yin JJ, Natale DA: A comprehensive evolutionary classification of proteins encoded in complete eukaryotic genomes. Genome Biol 2004, 5:R7.

25. Li XB, Zhang ML, Cui HR: Data mining for SSRs in ESTs and development of EST- SSR marker in oilseed rape. J Mol Cell Biol 2007, 40:137 144

26. Li $X$, Cui $H$, Zhang M: Detecting the genetic diversity of Brassica napus by EST-SSRs. J Agric Biotechnol 2007, 15:661 667.

27. Li ZL, Jakkula RS, Hussey JP, Boerma HR: SSR mapping and confirmation of the QTL from PI96354 conditioning soybean resistance to southern root-knot nematode. Theor App/ Genet 2001, 103:1167 1173

28. Nei M, Takezaki N: Estimation of genetic distances and phylogenetic trees from DNA anlysis. In Proc 5th World Cong Genet Appl Livstock Prod. 1983:405 412

29. Liu K, Muse SV: PowerMarker: an integrated analysis environment for genetic marker analysis. Bioinformatics 2005, 21:2128 2129.

30. Tamura K, Dudley J, Nei M, Kumar S: MEGA4: molecular evolutionary genetics analysis (MEGA) software version 4.0. Mol Biol Evol 2007, 24:1596 1599

31. Pritchard JK, Stephens M, Donnelly P: Inference of population structure using multilocus genotype data. Genetics 2000, 155:945 959.

32. Li X, Yan W, Agrama H, Hu B, Jia L, Jia M, Jackson A, Moldenhauer K, Mcclung A, Wu D: Genotypic and phenotypic characterization of genetic differentiation and diversity in the USDA rice mini-core collection. Genetica 2010, 138:1221 1230.

33. Rohlf FJ: NTSYS-pc: numerical taxonomy and multivariate analysis system, version 2.1. 2000

34. Li X, Yan W, Agrama H, Jia L, Shen X, Jackson A, Moldenhauer K, Yeater K, Mcclung A, Wu D: Mapping QTLs for improving grain yield using the USDA rice mini-core collection. Planta 2011, 234:347 361.
35. Schneider S, Excoffier L: Estimation of past demographic parameters from the distribution of pairwise differences when the mutation rates vary among sites: application to human mitochondrial DNA. Genetics 1999, 152:1079 1089 .

36. Gur-Arie R, Cohen CJ, Eitan Y, Shelef L, Hallerman EM, Kashi Y: Simple sequence repeats in Escherichia coli: abundance distribution, composition, and polymorphism. Genome Res 2000, 10:62 71.

37. Zalapa JE, Cuevas H, Zhu H, Steffan S, Senalik D, Zeldin E, McCown B, Harbut R, Simon P: Using next-generation sequencing approaches to isolate simple sequence repeat (SSR) loci in the plant sciences. Am J Bot 2012, 99(2):193 208.

38. Scott KD, Eggler P, Seaton G, Rossetto M, Ablett EM, Lee LS, Henry RJ: Analysis of SSRs derived from grape ESTs. TAG Theor Appl Genet 2000, 100(5):723 726.

39. Thiel T, Michalek W, Varshney RK, Graner A: Exploiting EST databases for the development of cDNA derived microsatellite markers in barley (Hordeum vulgare L.). Theor App/ Genet 2003, 106:411 422

40. Gupta PK, Rustgi S, Sharma S, Singh R, Kumar N, Balyan HS: Transferable EST-SSR markers for the study of polymorphism and genetic diversity in bread wheat. Mol Genet Genomics 2003, 270(4):315 323.

41. Huang Y, Li F, Chen K: Analysis of diversity and relationships among Chinese orchid cultivars using EST-SSR markers. Biochem Syst Ecol 2010, 38:93 102.

42. Wang HZ, Wu ZX, Lu JJ, Shi NN, Zhao Y, Zhang ZT, Liu JJ: Molecular diversity and relationships among Cymbidium goeringii cultivars based on inter-simple sequence repeat (ISSR) markers. Genetica 2009, 136:391 399.

43. Wang HZ, Wang YD, Zhou XY, Ying QC, Zheng KL: Analysis of genetic diversity of 14 species of Cymbidium based on RAPDs and AFLPs. Acta Biologiae Exp Sinica 2004, 37:482 486

44. Zhang JX, Li ZL, Fan CM, Cheng SL, Zhao MF, He YQ: Phylogenetics analysis of the Chinese orchids in Yunnan province using AFLP technique. Acta Horticulturae Sinica 2006, 33:1141 1144.

45. Woodhead M, Russell J, Squirrell J: Comparative analysis of population genetic structure in Athyrium distentifolium (Pteridophyta) using AFLPs and SSRs from anonymous and transcribed gene regions. Mol Ecol 2005 14:1681 1695

46. Clark AG, Glanowski S, Nielsen R, Thomas PD, Kejariwal A, Todd MA, Tanenbaum DM, Civello D, Lu F, Murphy B, Ferriera S, Wang G, Zheng X, White TJ, Sninsky JJ, Adams MD, Cargill M: Inferring non-neutral evolution from human-chimp-mouse orthologous gene trios. Science 2003, 302:1960 1963.

47. Tiffin P, Hahn MW: Coding sequence divergence between two closely related plant species: Arabidopsis thaliana and Brassica rapa ssp pekinensis. $\mathrm{J}$ Mol Evol 2002, 54:746 753

48. Kimura M: The neutral theory of molecular evolution: a review of recent evidence. Jpn J Genet 1991, 66(4):367 386

49. Nei M: Selectionism and neutralism in molecular evolution. Mol Biol Evol 2005, 22(12):2318 2342.

50. Griffiths S, Dunford RP, Coupland G, Laurie DA: The evolution of CONSTANS-like gene families in barley, rice, and Arabidopsis. Plant Physiol 2003, 131(4):1855 1867.

51. Fujiwara T, Mitsuya S, Miyake $H$, Hattori T, Takabe $T$ : Characterization of a novel glycinebetaine/proline transporter gene expressed in the mestome sheath and lateral root cap cells in barley. Planta 2010, 232(1):133 143

52. Grallath S, Weimar T, Meyer A, Gumy C, Suter-Grotemeyer M, Neuhaus JM, Rentsch D: The AtProT family. Compatible solute transporters with similar substrate specificity but differential expression patterns. Plant Physiol 2005, 137:117 126

53. Schwacke R, Grallath S, Breitkreuz KE, Stransky E, Stransky H, Frommer WB, Rentsch D: LeProT1, a transporter for proline, glycine betaine, and gamma-amino butyric acid in tomato pollen. Plant Cell 1999, 11:377 392

54. Igarashi Y, Yoshiba Y, Takeshita T, Nomura S, Otomo J, Yamaguchi-Shinozaki K, Shinozaki K: Molecular cloning and characterization of a cDNA encoding proline transporter in rice. Plant Cell Physiol 2000, 41(6):750 756.

55. Ueda A, Shi W, Sanmiya K, Shono M, Takabe T: Functional analysis of salt-inducible proline transporter of barley roots. Plant Cell Physiol 2001, 42(11):1282 1289. 
56. Hamel LP, Nicole MC, Duplessis S, Ellis BE: Mitogen-activated protein kinase signaling in plant-interacting fungi: distinct messages from conserved messengers. Plant Cell 2012, 24(4):1327 1351.

57. Pitzschke A, Schikora A, Hirt H: MAPK cascade signalling networks in plant defence. Curr Opin Plant Biol 2009, 12(4):421 426.

58. Andreasson E, Ellis B: Convergence and specificity in the Arabidopsis MAPK nexus. Trends Plant Sci 2010, 15(2):106 113.

doi:10.1186/s12863-014-0124-5

Cite this article as: Li et al:: Development of Cymbidium ensifolium

genic-SSR markers and their utility in genetic diversity and population

structure analysis in cymbidiums. BMC Genetics 2014 15:124.

\section{Submit your next manuscript to BioMed Central and take full advantage of:}

$\otimes$ Convenient online submission

$\otimes$ Thorough peer review

$\nabla$ No space constraints or color $\nabla$ gure charges

$\otimes I m m e d i a t e$ publication on acceptance

$\triangle$ Inclusion in PubMed, CAS, Scopus and Google Scholar

$\otimes$ Research which is freely available for redistribution 